\title{
Late recurrence of Castleman's disease with mixed angiomyoid, histiocytic reticulum cell, follicular dendritic cell stroma-rich proliferations: a case report and review of the literature
}

\author{
Raquel Walsh-Jahnke $\cdot$ Wei Cui $\cdot$ Da Zhang
}

Received: 3 November 2014 / Accepted: 12 February 2015 / Published online: 26 February 2015

(C) Springer-Verlag Berlin Heidelberg 2015

\begin{abstract}
Castleman disease, hyaline vascular variant (HV$\mathrm{CD}$ ) involving lymph nodes is characterized by abnormal follicles with increased vascularity, hypervascular interfollicular tissue, absence of sinuses, and follicles having the characteristic "onion skin-like" appearance. We present a rare case of HV-CD, stroma-rich variant, which recurred less than 5 years after initial surgical therapy. The recurrent lesion varied in appearance from classical morphology of HV-CD with a stromal proliferation of follicular dendritic, angiomyoid cells, and histiocytic reticulum cells. Less than fifty cases of stroma-rich Castleman disease have been reported in the literature. To the best of our knowledge, this is the first case showing a mixed proliferation of stromal elements with rims of compressed normal lymphoid tissue and recurrence after surgical excision.
\end{abstract}

Keywords Castleman disease $\cdot$ Hyaline vascular type . Stroma-rich variant

\section{Introduction}

Castleman disease (CD) was first described in 1956 as a large, benign mass involving the mediastinal lymph nodes and mimicking thymoma. [1] Two different histologic patterns have

\section{R. Walsh-Jahnke $\cdot$ W. Cui $\cdot$ D. Zhang $(\bowtie)$}

Department of Pathology and Laboratory Medicine, Kansas

University Medical Center, 3901 Rainbow Boulevard, Kansas

City, KS 66160, USA

e-mail: dzhang@kumc.edu

R. Walsh-Jahnke

e-mail: rwalsh@kumc.edu

W. Cui

e-mail:wcui@kumc.edu since been described: the hyaline vascular (HV) and plasma cell (PC) variant Castleman disease. The HV variant is more common, typically presents as a solitary lesion involving the cervical or mediastinal lymph nodes and is asymptomatic. This variant represents the majority of all cases and is typically a disease of young adults. It is characterized by small follicles of lymphocytes arranged around a hyalinized blood vessel with prominent interfollicular capillary proliferations and mantle zones of the follicles arranged with an onion skinlike pattern often described as having a "lollipop" appearance. The second histologic type is PC variant which is often symptomatic with fever, splenomegaly and is multicentric.

Angiomyoid foci and proliferation of stromal and follicular dendritic cells have been described in HV-CD with overgrowth of myoid cells, follicular dendritic cells, or dendritic histiocytes, with or without an increase in the number of blood vessels [2]. If the interfollicular abnormality accounts for more than $50 \%$ of the lymph node, it is defined as stroma-rich variant [2]. To date, there have been less than 50 cases $[2,3,7,8]$ of stroma-rich variant of HV-CD reported in the literature; more than half of the cases are from two studies performed by the same author [2, 3]. However, to the best of our knowledge, no cases have been reported which include follicular dendritic and angiomyoid proliferations within the same lesion and demonstrate a rim of residual normal lymphoid tissue.

We present a case of HV-CD, with a stromal proliferation composed of mixed follicular dendritic cells, histiocytic reticulum cells, and myoid cells which recurred less than 5 years following initial surgical management and a review of the literature.

Clinical history

A 26-year-old white female presented in September 2008 with intermittent lower pelvic pain. She had recently started Nuva 
Ring for contraception and denied irregular menstrual bleeding or weight fluctuation. A serum pregnancy test for human chorionic gonadotropin (HCG) was negative and CEA and CA-125 levels were within normal limits. At that time, a computed tomography scan (CT) was performed and showed a rounded, well-demarcated, solid homogenous mass in the left posterior adnexa measuring $8.1 \times 5.2 \mathrm{~cm}$ in diameter. No other mass or lymphadenopathy is identified. The patient underwent excision of the mass in November 2008 and pathologic evaluation revealed a $\mathrm{CD}$, $\mathrm{HV}$ variant with an extensive proliferation of stromal cells. No further treatment was rendered.

At a routine primary care office visit in February 2013, a mass was palpated in the left upper quadrant of the abdomen that was tender to palpation. A CT exam showed a large periaortic soft tissue mass at the bifurcation of the aorta which measured $9.5 \times$ $4.5 \times 4.5 \mathrm{~cm}$. The patient was referred to our institution for further management, and complete surgical excision of the mass was performed with a diagnosis of recurrent $\mathrm{CD}$.

\section{Materials and methods}

Formalin-fixed (10\% neutral, buffered) paraffin embedded sections were stained by routine hematoxylin and eosin preparations. Immunohistochemical analysis was performed on Ventana automated machine from selected blocks. The panel of reagents used included antibodies to B cells (CD20), T cells (CD1a, CD2, CD3, CD4, CD5, CD7, CD8, CD43), follicular dendritic cells (CD21, CD35), histiocytes and plasmacytoid monocytes (CD68, CD163, lysozyme), interdigitating cells (S-100), endothelia (CD34, CD31), myoid cells (antismooth muscle, desmin), melanocytic cells (HMB-45), hematopoietic precursors (myeloperoxidase, CD45 LCA, CD10, CD15), cytokeratin marker (Pan-CK), tumor suppressor gene mutation marker (p53), viral stains (immunohistochemical stain for HHV8, in situ hybridization for EBV), and a marker of proliferative pattern (MIB-1). The vendors of these antibodies and their clones are Dako (CD1a 010, CD2 AB75, CD7 CBC.37, CD35 Ber-MAC-DRC, MIB-1 Ki-67), Ventana (CD3 2GV6, CD4 SP35, CD5 SP19, CD8 SP57, CD15 MMA, CD20 L26, CD30 Ber H2, CD34 QBEnd/10, CD45LCA RP2/18, ALK-1 ALK01, HMB45 HMB-45, Lysozyme poly, S100 poly, Desmin DER-11, CD68 KP-1, PanCK AE1/AE3/PCK26, EBV probe), LabVision (CD163 10D6), BioCare (CD21 2G9), and CellMarque (CD10 56C6, CD123 6H6, Myeloperoxidase poly, HHV8 13B10). All immunohistochemical stains follow the manufacturer's protocol.

\section{Results}

The surgical specimen consisted of an oval-shaped mass with a smooth surface which measured $14 \times 7 \times 6 \mathrm{~cm}$. Cross-section analysis showed a well-encapsulated, homogenous, firm pinktan mass which had central fibrosis. Hematoxylin-eosinstained sections showed a lymph node with numerous regressive lymphoid follicles, expanded interfollicular regions composed of sclerotic blood vessels, plump spindle cells, and fibrosis with a thick fibrous capsule. The interfollicular abnormalities represented more than $50 \%$ of the lymph node. Rims of residual normal lymphoid tissue were seen in some sections (Fig. 1a). Occasional follicles contained radial arterioles with sclerotic walls and onion-skinning mantle zone imparting a hyaline vascular appearance (Fig. 1b). One of the more prominent features was nodular proliferation of histiocytic reticulum cells (Fig. 1c) within the residual germinal centers and stroma, highlighted by CD68 (Fig. 1d), myoid cells between the lymphoid follicles (Fig. 1e), highlighted by smooth muscle actin (SMA) (Fig. 1f), and follicular dendritic cells (Fig. 1g). These vague nodules had a heterogeneous cellular composition with plump, oval to spindle cells with vesicular nuclei and admixed with small lymphocytes (Fig. $1 \mathrm{~b}$ inset). Mitoses were inconspicuous with no significant atypia. The previous resection specimen was reviewed; it showed a similar morphology with prominent stromal proliferation and vague nodule formation. However, no residual normal lymphoid tissue was identified on the submitted specimen.

Angiomyoid proliferation composed only a small portion of the nodular proliferations after histiocytic reticulum cells and follicular dendritic cells. The anti-CD21 and CD35 stains highlighted atrophic germinal centers as well as the normal follicular dendritic framework within the germinal centers (Fig. 1h). The stroma is mostly negative for CD21 and $\mathrm{CD} 35$, but within the nodular areas, these stains are focally increased. The anti-CD68 and anti-CD163 antibodies highlighted many histiocytic reticulum cells in nodules within the stroma. These cells were particularly prominent within the myoid proliferation nodules. HHV8 stain was negative. In situ hybridization for EBV using EBER stain was negative. MIB-1 stain demonstrated a relatively low proliferation rate throughout the lesion, less than $5 \%$ within follicles and approximately 5-10\% within the stromal areas. The follicular dendritic proliferation and histiocytic reticulum cell proliferation nodules show a higher MIB-1 rate of $10-20 \%$. CD123 positive plasmacytoid cells are scattered in the regressive/atrophic lymphoid follicles, the residual normal lymphoid tissue, and proliferative stromal areas but do not form aggregates. The results with antibodies specific for CD1a, CD10, ALK-1, p53, HMB-45, pan-cytokeratin, CD30, and CD15 were negative. S-100 stain highlighted scattered background interdigitating reticulum cells; no aggregates of positive cells were identified. The germinal centers are composed predominantly of B cells that are positive for CD20 with scattered T cells positive for $\mathrm{CD} 3$ in the regressive/atrophic lymphoid follicles, the residual normal lymphoid tissue and proliferative stromal areas. 
Fig 1 Representative section of mass with atrophic germinal centers with proliferation of interfollicular areas and rim of residual normal lymphoid tissue (a). Small follicles of lymphocytes arranged around a hyalinized blood vessel with prominent interfollicular capillary and stromal proliferations showed a "lollipop" appearance (b). The adjacent stroma cells are plump, oval to spindle cells with vesicular nuclei and admixed with small lymphocytes (B inset).

Histiocytic reticulum cell nodular proliferation in residual germinal center (c) highlighted by immunohistochemical stain for CD68 (d). Angiomyoid proliferation (e) within the stroma is highlighted by immunohistochemical stain for SMA (f). Follicular dendritic cell proliferation outside a residual germinal center $(\mathbf{g})$ and immunohistochemical stain for CD21 highlights two atrophic germinal centers as well as the residual follicular dendritic framework $(\mathrm{H})$
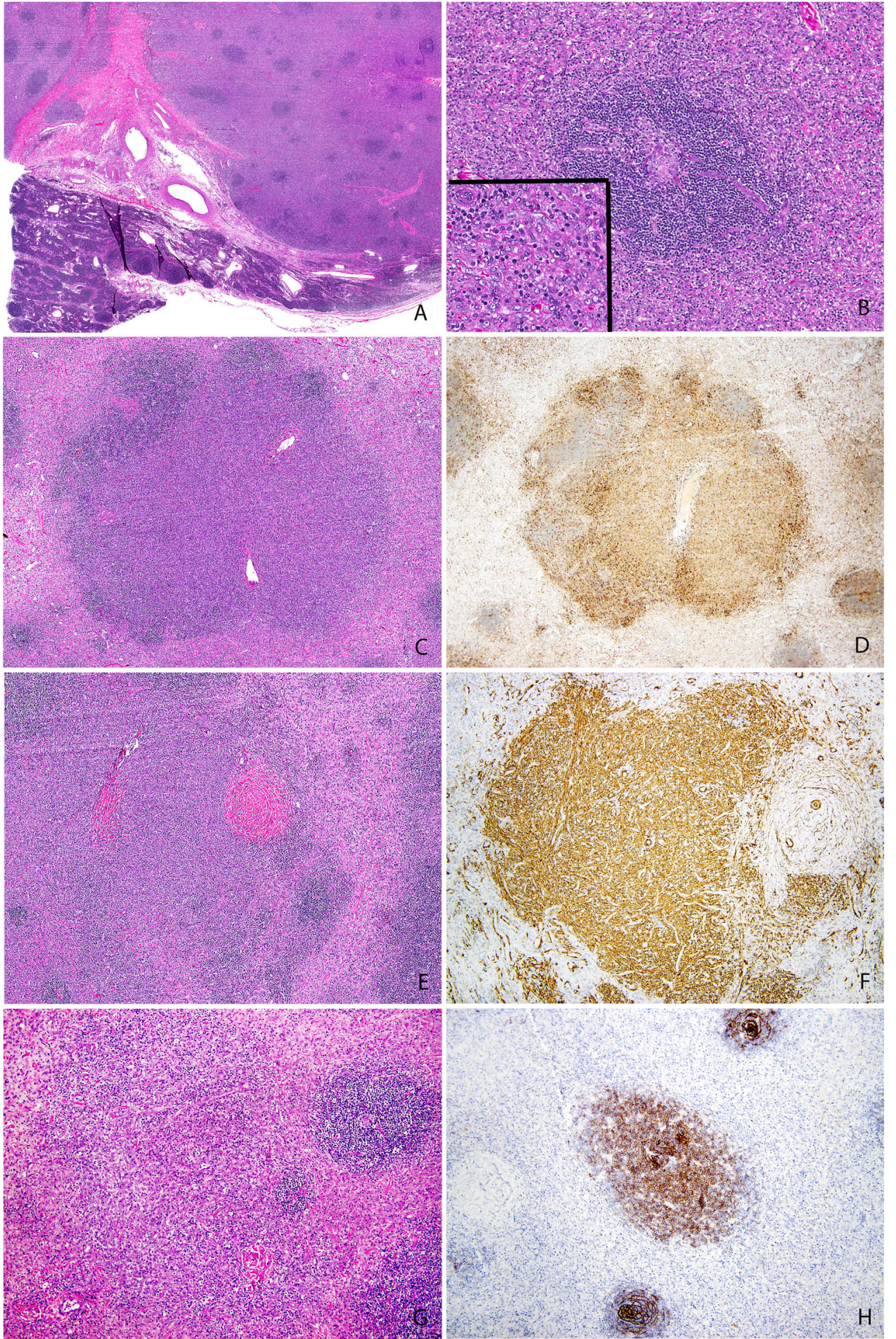

The clinical profile, morphology, and immunohistochemical studies were consistent with a diagnosis of CD, stromarich with spectrum of morphology from classic $\mathrm{HV}$ variant to follicular dendritic cell, histiocytic reticulum cell, and angiomyoid proliferations.

\section{Discussion}

Two major variants of CD have been described, including the $\mathrm{HV}$ and $\mathrm{PC}$ types with the former comprising most of the cases (90\%) [4]. For the purposes of this discussion, we will be 
focusing on the HV type. The HV type occurs over a wide age range with no sex predilection. It typically presents as a large mass involving a lymph node and typically will only cause symptoms if the mass obstructs major organs or structures. Other laboratory abnormalities are rare [4].

HV-CD is prone to stromal overgrowths, in which the stromal component comprises greater than $50 \%$ of the total lymph node, with angiomyoid, interfollicular, histiocytic, follicular dendritic cell, and vascular proliferations all being reported. [1, 2]. Identification of these stroma-rich $\mathrm{CD}$ is important since association between follicular dendritic overgrowth in HV-CD and subsequent developing sarcoma including follicular dendritic sarcoma were reported $[2,6]$. Some studies have shown a relationship between follicular dendritic cell sarcoma and HV-CD with increased epidermal growth factor receptor (EGFR) expression in the tumor cells of follicular dendritic cell (FDC) sarcoma and all types of CD that was absent in the FDC's of reactive germinal centers in other lymphomas. It is postulated that upregulation of EGFR promotes FDC persistence, allowing subsequent accumulation of mutations that may result in the infrequent emergence of sarcoma [12]. However, the etiology of HV-CD still remains unclear.

Some authors have even thought that the Castleman-like phenomenon could be the reactive feature in some of these cases; however, stromal cell clonal proliferation has been reported [9-11]. In cases of HV-CD with a proliferation of stromal cells, several other entities must be ruled out including Hodgkin lymphoma, follicular dendritic cell tumor/neoplasm when cytological atypia and mitosis are present, and Kaposi's sarcoma (HHV8 associated).

To further characterize the stromal proliferation, a panel of immunohistochemical stains is recommended. The follicular dendritic cell proliferative variant appears to be the only one with neoplastic potential, whereas the others tend to be more hyperplastic and indolent in their clinical course. The cases with predominance of angiomyoid overgrowth are less likely to recur or metastasize than cases with follicular dendritic cell proliferation [2].

The common site for stroma-rich cases has been the retroperitoneum $[1,2,6]$, most likely due to the deep location and space for tumor to grow large sizes before causing symptoms. Cases with isolated angiomyoid proliferations most often occur in young female patients are more likely to occur in the retroperitoneum, typically manifest as slow growing lesions, and are considered hyperplastic as opposed to neoplastic. Most of these lesions are cured with complete surgical excision. No other treatments are typically recommended.

The clinical scenario in cases with follicular dendritic cell proliferations are quite different from the angiomyoid types as there have been reports showing clonality within the follicular dendritic cell proliferations [6] and are considered to be premalignancy or neoplastic. These have no sex predilection, occur in older patients and in a broad spectrum of locations, and are more likely to recur. Currently, CD with follicular dendritic cell proliferations is treated by surgical excision only. However, due to the neoplastic nature of the disease, other therapeutic options may be considered in patient's refractory to surgical intervention.

The present case is unique in the fact that it possessed a spectrum of morphology from classic "lollipop" appearance, to follicular dendritic, histiocytic reticulum cell and angiomyoid cell proliferation, residual normal lymphoid tissue, and recurred less than 5 years after initial surgical management. To the best of our knowledge, there has been only one other reported case of $\mathrm{CD}$, stroma-rich with a mixed histologic type [5] and our case is the only case that showed rim of residual normal lymphoid tissue. The lack of cellular atypia, mitosis, significant MIB-1 positivity, and the wellcircumscribed nature of the tumor in the present case argued against a sarcomatous lesion. There are two possible explanations for the appearance of the recurrent lesion. One is metastasis, with a clonal population of cells draining into the adjacent lymph node and expanding. The other possibility is de novo, where the tumor cells expand and push the normal tissue to the edge. As the recurrent lesion is adjacent to the previous site and there is a rim of residual normal lymphoid tissue, we favor the first possibility. The 5-year interval between the first and the second lesion indicates the indolent nature of the neoplasm.

The classic CD is rarely found in such large size probably due to their earlier detection and excision. The stroma-rich variant of HV-CD is commonly found in the retroperitoneal space where the lesion is difficult to detect, allowing it to grow large and present at a late stage. $\mathrm{CD}$ has been associated with follicular dendritic cell tumor/neoplasm. We believe classic HV-CD, stroma-rich variant with angiomyoid, or follicular dendritic proliferation and follicular dendritic cell tumor/ neoplasm represent a spectrum of disease at different stages. Because of the uncertainty of the nature of this condition and its potential progression to a more aggressive entity, patients with stroma variant $\mathrm{CD}$ should be followed more closely by their surgeons and oncologists to evaluate for recurrence or metastasis.

Conflict of interest The authors declare that they have no conflict of interest.

\section{References}

1. Castleman B, Iverson, Menendez VP (1956) Cancer. Localized mediastinal lymph node hyperplasia resembling thymoma. 9[4]:822-30

2. Lin O, Frizzera G (1997) Angiomyoid and follicular dendritic cell proliferative lesions in Castleman's disease of hyaline-vascular type: a study of 10 cases. Am J Surg Pathol 21(11):1295-306 
3. Danon AD, Krishnan J, Frizzera G (1993) Morphoimmunophenotypic diversity of Castleman's disease, hyalinevascular type: with emphasis on a stroma-rich variant and a new pathogenetic hypothesis. Virchows Arch A Pathol Anat Histopathol 423(5):369-82

4. Ioachim H ML. Castleman Lymphadenopathy. Lymph node Pathology, 4th edition:227-37

5. Chan JK, Tsang WY, Ng CS (1994) Follicular dendritic cell tumor and vascular neoplasm complicating hyaline-vascular Castleman's disease. Am J Surg Pathol 18(5):517-25

6. Chan AC, Chan KW, Chan JK, Au WY, Ho WK, Ng WM (2001) Development of follicular dendritic cell sarcoma in hyaline-vascular Castleman's disease of the nasopharynx: tracing its evolution by sequential biopsies. Histopathology 38(6): $510-8$

7. Izumi M, Mochizuki M, Kuroda M, Iwaya K, Mukai K (2002) Angiomyoid proliferative lesion: an unusual stroma-rich variant of Castleman's disease of hyaline-vascular type. Virchows Arch 441(4): $400-5$
8. Xiong Y, Zhang Y, Wang Y, Na J, Li T (2010) Stroma-rich variant of hyaline-vascular Castleman's disease: a clinicopathologic and histogenetic study. Zhonghua Bing Li Xue Za Zhi 39(1):14-8

9. Chen WC, Jones D, Ho CL, Cheng CN, Tseng JY, Tsai HP, Chang KC (2006) Cytogenetic anomalies in hyaline vascular Castleman disease: report of two cases with reappraisal of histogenesis. Cancer Genet Cytogenet 164(2):110-7

10. Cokelaere K, Debiec-Rychter M, De Wolf-Peeters C, Hagemeijer A, Sciot R (2002) Hyaline vascular Castleman's disease with HMGIC rearrangement in follicular dendritic cells: molecular evidence of mesenchymal tumorigenesis. Am J Surg Pathol 26(5):662-9

11. Pauwels P, Dal Cin P, Vlasveld LT, Aleva RM, van Erp WF, Jones D (2000) A chromosomal abnormality in hyaline vascular Castleman's disease: evidence for clonal proliferation of dysplastic stromal cells. Am J Surg Pathol 24(6):882-8

12. Sun X, Chang KC, Abruzzo LV, Lai R, Younes A, Jones D (2003) Epidermal growth factor receptor expression in follicular dendritic cells: a shared feature of follicular dendritic cell sarcoma and Castleman's disease. Hum Pathol 34(9):835-40 